\title{
Neglected patellar tendon rupture: a case of reconstruction without quadriceps lengthening
}

\author{
D. Bek • B. Demiralp • M. Kömürcü • A. Şehirlioğlu
}

Received: 3 July 2007 / Accepted: 4 January 2008 / Published online: 17 March 2008

\begin{abstract}
Neglected rupture of the patellar tendon is a rare, can be easily missed in a group of patients. We present a 24 year old, male patient who sustained right femoral diaphyseal and tibial plateau fractures and a patellar tendon rupture following a motor vehicle accident. The fractures were treated by open reduction internal fixation but the patellar tendon rupture was missed and the diagnosis was delayed by 7 months. Patella was migrated proximally. It was moved distally to the original location and neglected patellar tendon rupture treated successfully with modified Ecker technique. Neither preoperative traction nor additional intraoperative procedures were performed to relocate the patella to its anatomic position in the extended knee and good functional result was achieved with intensive rehabilitation.
\end{abstract}

Keywords Neglected patellar tendon rupture · Late surgical repair $\cdot$ Sequential bracing

D. Bek (匈) · B. Demiralp · M. Kömürcü · A. Şehirlioğlu

Department of Orthopaedics and Traumatology

Gülhane Military Medical Academy

06018 Ankara, Turkey

e-mail: doganbek@hotmail.com

\section{Introduction}

Surgical management of neglected patellar tendon rupture is more demanding than that of acute ruptures, and the results are less favorable because of retraction, adhesion, atrophy of the quadriceps muscle and proximal patellar migration [1-3]. There are several methods to relocate the patella to its anatomic location including preoperative traction, intraoperative traction, external fixation and quadricepsplasty [1-8]. We present a patient with a neglected patellar tendon rupture treated successfully with a modified Ecker technique [7] and adjustable knee brace in the postoperative period. Neither preoperative traction nor additional intraoperative procedures were performed to relocate the patella to its anatomic position in the extended knee. Good functional result was achieved with intensive rehabilitation.

\section{Case report}

A 24-year-old man was admitted to our clinic with a complaint of inability to extend his right knee. His medical history revealed a motor vehicle accident 7 months earlier, in which he sustained right-sided ipsilateral femoral diaphyseal fracture and tibial plateau fracture, and hemothorax. Intramedullary nail fixation for the femoral fracture and open reduction and cannulated screw fixation for the tibial plateau fracture had been performed at another institution.

On physical examination, an anterolateral inverted Jshaped incision was remarkable over his right knee and the patella was migrated proximally. The passive range of motion (ROM) of his right knee was normal; however, there was no active knee extension. Radiographs revealed 


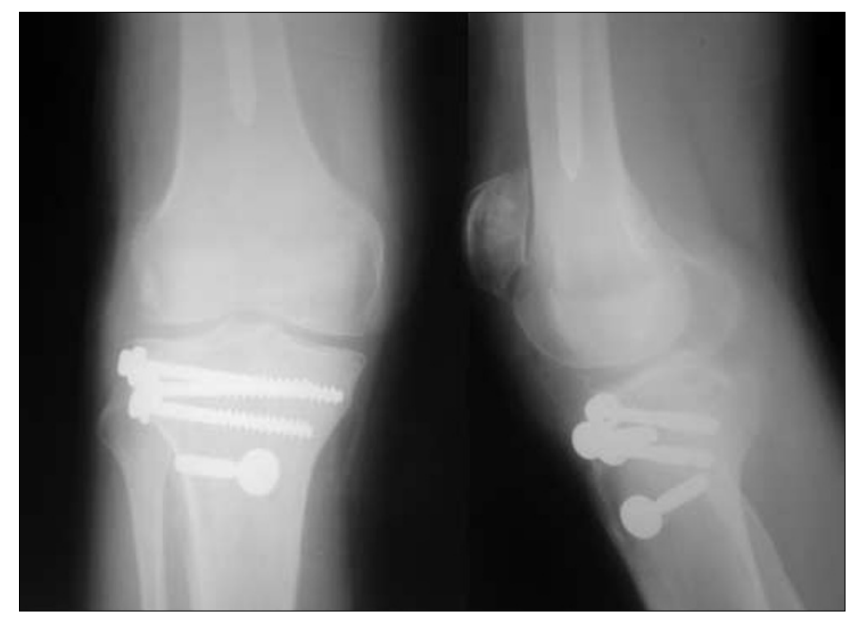

Fig. 1 Preoperative anteroposterior (left) and lateral (right) knee radiographs. Lateral view shows elevated patella

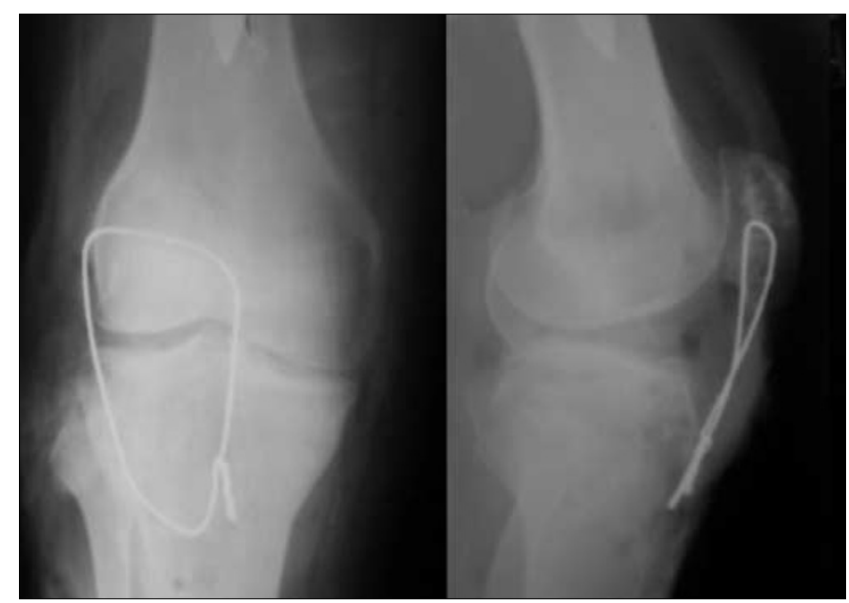

Fig. 2 Postoperative anteroposterior (left) and lateral (right) knee radiographs. Lateral view shows anatomic location of the patella

fracture union in both the femur and tibial plateau and high location of patella (Fig. 1). With all these clinical and radiographic findings, we diagnosed neglected patellar tendon rupture.

Surgical treatment was planned. An anterior midline skin incision was used. Flaps were dissected to expose the patella and tibial tuberosity. Scar tissue in the remnants of the patellar tendon was exiced. One transverse tunnel in the patella and one in tibial tuberosity were drilled after removal of the cannulated screws. While the knee was held in extension, a circular wire was passed from the tunnels and tightened until obtaining an adequate distance between the patella and the tibial tuberosity, considering the Insall-Salvati ratio (normal length of the patellar tendon is approximately equal to the length of the patella) [9]. There was no difficulty to pull down the patella by tightening the cerclage wire (Fig. 2) [1, 10]. Apart from the Ecker technique, we did not use a Steinman pin for patellar traction and only one hole was drilled to the patella for semitendinosus-gracilis tendons and cerclage wire
[7]. Remnants of the patellar tendon were repaired with primary sutures as far as we could. The ends of the semitendinosus and gracillis tendons were exposed at their insertion and harvested by the open-end tendon stripper from their proximal musculotendinous junction and brought into the primary incision. The semitendinosus tendon was passed through the tunnel drilled in the tibial tuberosity and through the tunnel drilled in the patella and sutured to the gracilis tendon. Then, the gracilis tendon was passed through the same tunnel drilled in the patella too and sutured to the semitendinosus tendon (Fig. 3). After the repair was completed, when the hip was flexed at $45^{\circ}$, the knee could be flexed to $20^{\circ}$ with gravity of the leg. At this point the repair was stable and the knee was immobilized by the plaster cast in $20^{\circ}$ flexion at the end of the procedure.

Cast and sutures were removed after 2 weeks. Continuous passive motion (CPM) was applied for the next 3 days, beginning at $0^{\circ}-20^{\circ}$ with close monitoring and reaching $30^{\circ}$ of knee flexion at the end of the third day.

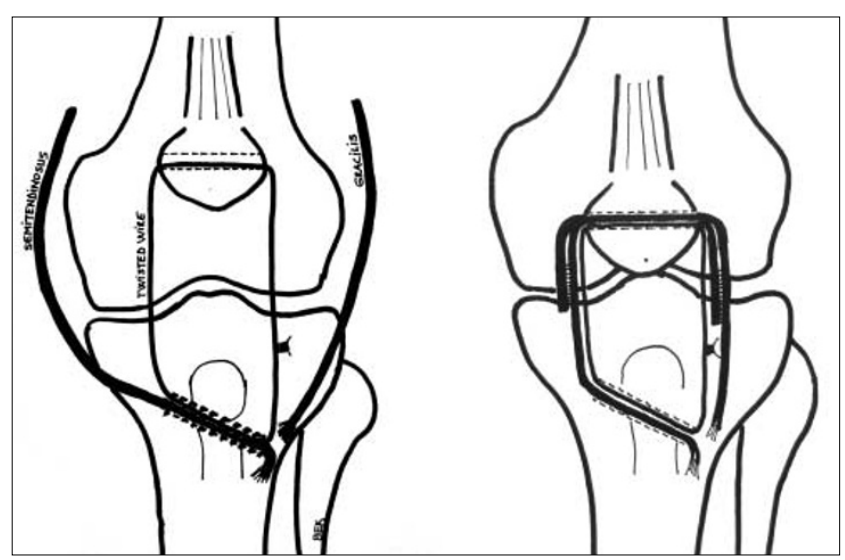

Fig. 3 Reconstruction of the patellar tendon with semitendinosus and gracilis tendons after relocation of the patella by twisted wire

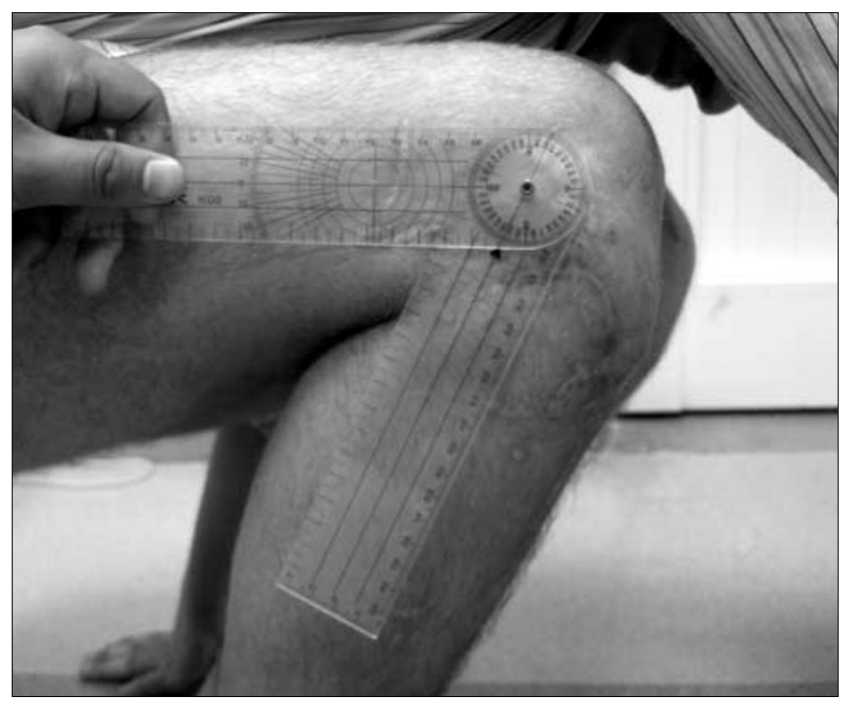

Fig. 4 Flexion of right knee after 2 years 
An adjustable brace was applied with the maximum flexion set at $30^{\circ}$. Daily ROM exercises were performed within the range of previously reached ROM, three times a day until the end of the 2-week period. This protocol was repeated at the fourth and sixth weeks postoperatively, and $50^{\circ}$ and $70^{\circ}$ of flexion were reached, respectively. The brace was removed at eight weeks and CPM and ROM exercises were continued until the tenth week postoperatively. Finally, $110^{\circ}$ of flexion was achieved (Fig. 4). Perfect recovery of the extensor unit of the knee was obtained and the patient returned to work as a technician five months after surgery. Five degrees of extension lag persisted as a sequela and quadriceps muscle strength reached just $15 \%$ less than the contralateral extremity, as confirmed by Cybex testing after two years.

\section{Discussion}

Fresh ruptures of the patellar tendon, which usually occur at the inferior pole of the patella, require immediate surgical restoration of the extensor mechanism for optimal return to preinjury functional status. End-to-end repair is used with or without a reinforcing cerclage suture of wire or nonabsorbable suture material or, alternatively, tape and cast immobilization is recommended for 6-8 weeks postoperatively [3]. Better results have been reported in immediate repairs of fresh patellar tendon ruptures in terms of ROM, the strength of the quadriceps muscle and overall functional results [3]. Neglected rupture of the patellar tendon is a rare condition [3, 5-8]. Even if the proper physical examination is performed, patellar tendon rupture can be easily missed in patients with multitrauma, obesity and knee hemathrosis $[3,8]$. Simple reapproximation of the torn tendon ends is often difficult when repair has been delayed more than six weeks. The longer the delay between injury and repair, the greater the likelihood of quadriceps retraction and proximal patellar migration. Fibrous adhesions may develop between the patella and the underlying femur. The ruptured tendon ends shrivel and become encased in scar tissue. Primary repair combined with autogenous graft augmentation using fascia lata or hamstring tendons has been most commonly used [11]. Reconstruction with extensor mechanism allografts consisting of an Achilles tendon or an intact patellar tendon has also been used as a salvage procedure [12]. Many techniques have been reported to relocate the patella to its anatomic position and to repair the patellar tendon; however, there is no widely accepted method. External fixation using pins and wires has been reported as a solution for patients with severe contracture of the quadriceps tendon and elevated patella [1, 3, 4, 6]. The Ilizarov method has also been used to relocate the patella to its original

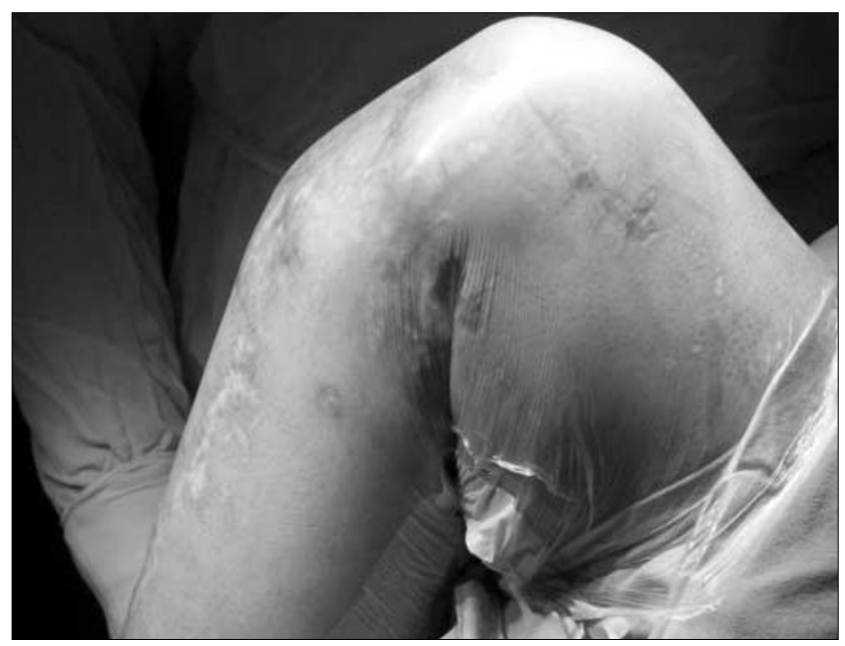

Fig. 5 Obvious elevation of the patella in knee flexion before the incision

anatomic position [5]. Mandelbaum et al. [2] recommended $\mathrm{Z}$ lengthening for the quadriceps tendon and $\mathrm{Z}$ shortening for the patellar tendon with augmentation using the semitendinosus and gracilis tendons. They applied a cylinder cast for the first two weeks with the knee in full extension and progressively increased the amount of flexion (10 degrees/week) in a hinged knee brace. They reached $90^{\circ}$ of active flexion at 10 weeks and $130^{\circ}$ at the one-year follow-up.

Although proximal migration and gap in our case became obvious with knee flexion (Fig. 5), patellar migration did not seem to be pronounced in the extended knee. Neither preoperative traction nor additional intraoperative procedures were performed to relocate the patella to its anatomic position. We did not have any difficulty to move the patella distally by tightening the cerclage wire intraoperatively in the extended knee, as mentioned in the literature $[1,10]$. Tightening the wire too much in the extended knee may result in patella infera [3]. After repair of the extensor mechanism, resultant quadriceps tension and restriction of flexion were counteracted upon with the use of CPM and repeated bi-weekly brace adjustments in maximum flexion. Good functional result was achieved. The positive outcome in this case suggest that this approach should be studied as a possible solution to obtain proper patellar position and functional ROM.

\section{References}

1. Takebe K, Hirohata K (1985) Old rupture of the patellar tendon. Clin Orthop Relat Res 196:253-255

2. Mandelbaum BR, Bartolozzi A, Carney B (1988) A systematic approach to reconstruction of neglected tears of the patellar tendon. Clin Orthop Relat Res 235:268-271

3. Siwek CW, Rao JP (1981) Ruptures of the extensor mechanism of the knee joint. J Bone Joint Surg Am 63:932-937 
4. Nsouli AZ, Nsouli TA, Haidar R (1991) Late reconstruction of the patellar tendon: case report with a new method of repair. J Orthop Trauma 31:1319-1321

5. Isiklar ZU, Varner KE, Lindsey RW et al (1996) Late reconstruction of patellar ligament ruptures using Ilizarov external fixation. Clin Orthop Relat Res 322:174-178

6. Kelikian H, Riashi E, Gleason J (1957) Restoration of quadriceps function in neglected tear of the patellar tendon. Surg Gynecol Obstet 104:200-204

7. Ecker ML, Lotke PA, Glazer RM (1979) Late reconstruction of the patellar tendon. J Bone Joint Surg Am 61:884-886

8. Shephard GJ, Christodoulou L, Hegap AIA (1999) Neglected rup- ture of the patellar tendon. Arch Orthop Trauma Surg 119:241-242

9. Insall J, Salvati EA (1971) Patella position in the normal knee joint. Radiology 101:101-104

10. Casey MT, Tietjens BR (2001) Neglected ruptures of the patellar tendon, a case series of four patients. Am J Sports Med 29:457-460

11. Matava MJ (1996) Patellar tendon ruptures. J Am Acad Orthop Surg 4:287-296

12. Burks RT, Edelson RH (1994) Allograft reconstruction of the patellar ligament: a case report. J Bone Joint Surg Am 76:1077-1079 